\title{
Chinese and English Reviews of a Story about Teenagers' Struggles
}

A Multi-method Analysis of Cultural Differences in Narrative Interpretation

\author{
Shenglan TAN \\ Teaching Fellow, Harvard XMedia Lab, Harvard University Graduate School \\ of Education, Cambridge, MA, USA \\ shenglan.tan19950129@gmail.com
}

Haiqing HE | ORCID: 0000-0001-9918-6025

Research Assistant, Harvard XMedia Lab, Harvard University Graduate School of Education, Cambridge, MA, USA

hohoihelen@gmail.com

\section{Liu JIANG}

Ph.D. Candidate, Harvard University Graduate School of Education,

Cambridge, MA, USA

liujiang@fas.harvard.edu

\section{XUZHAO | ORCID: 0000-0001-7875-4863}

Director Research - Director of Research in Chinese Youth Mental Health and Well Being, Associate Professor, Werklund School of Education, University of Calgary, Calgary, Alberta, Canada xu.zhao@ucalgary.ca

\section{Robert L. SELMAN}

Professor of Psychology, Psychiatry Dept., Harvard Medical School, Graduate School of Education, Harvard University, Cambridge, MA, USA Robert_Selman@gse.harvard.edu

\begin{abstract}
Wonder, a fictional story told both in print (2/2012) and movie (11/2017) formats, depicts a middle-school student, Auggie, who struggles with social exclusion and being the target of school based relational bullying due to his genetically based facial deformity.
\end{abstract}


Internationally popular, abundant everyday reviews by non-professional commenter are easily accessible on-line, e.g., on Douban (Chinese) and Common-Sense Media (English). Access to these comments enables the investigation of what perspectives (knowledge and understanding, beliefs and opinions, etc.) reviewers draw upon in sharing their thoughts about the protagonist's struggles. In this study we undertook two analyses. In analysis 1, we asked, how do Chinese -/English -speaking reviewers comment on Auggie's struggles to be accepted by his peers at school, both the causes of his social problem and the ways Auggie dealt (or might deal) with his situation. In analysis 2, we explored comments that reviewers made about Auggie's only sibling, his elder sister, Via, a first-year high school students who, in the story, struggles to find a balance between meeting her own need for her parents' love and attention, and fulfilling her familial role and obligations, given Auggie's special needs.

Based on data from open-source "every day" books/movies review websites, we compared, culturally, the perspectives on the story expressed by Chinese and English commenters through emic (both thematic and discursive) exploratory analyses. Analysis 1 suggests that comparatively, Chinese-speaking reviewers tend to more often focus on the value of solving the problem, and in doing so, articulating the corresponding ways a victim of bullying or neglect can be or become both strong and interdependent, whereas English-speaking reviewers more often tend to emphasize exploring and understanding the causes of the relational problems and tend to suggest independenceoriented ways of solving the social problem by changing the actions of others. Analysis 2 suggests differences across Chinese- and English-speaking reviewers in perceptions of and judgements about the fundamental "culture based" determinants and ways for Via to deal with her relational struggle within the family.

\section{Keywords}

school bully - adolescent relational needs - storytelling - cultural comparison

\section{1 \\ Introduction}

Educators are constantly searching for and selecting humanistic stories to promote literacy, social and historical knowledge, and humanistic values such as sympathy to and acceptance of interpersonal differences as well as an openness to welcome newcomers to the community. But, generally speaking, we ask, what might be the various impacts on their intended audience academic, aesthetic, and ethical - of these stories so selected? And, how might an empirical analysis that includes a comparison between Mandarin 
and English-speaking commenters add to our understanding of how possible variations in humanistic values across cultures influence the way they make meaning of the story, such as how its plot and the actions of its characters are differentially or similarly interpreted? To demonstrate an empirical research approach to these daunting, multi-faceted questions we have undertaken an analysis of how on-line reviewers comment on and frame the plot and characters in one contemporary (American) story popular in both the United States and mainland China.

As background to our study we reviewed three domains of literature. What do we know about the social exclusion of newcomers in the peer culture of youth, especially in pre- and early adolescence, and of the reasons for their harassment by the members of the dominant peer group they seek to join? What do we know about variations in how youth from different cultures perceive the causes and coping devices to be used effectively in such circumstances of peer oriented social exclusion and family oriented "inattention"? What do we know about the positive impact of humanistic stories, mainly in print, but also in film, on readers' and viewers' social perspectives? We provide in our conclusion some general implications for the use of storytelling to elicit readers' and viewers' spontaneous comments on the topics of school bullying and family responses as they may have bearing on future research and potential practice, e.g., the use of cross media story-telling (Selman et al, 2020).

\section{$2 \quad$ Social Exclusion, The Case of Bullying in School}

Social exclusion has been broadly defined as the harmful experience of being isolated physically, socially, or emotionally (Riva \& Eck, 2016), with the effect to impair one's cognitive ability to process social information (Baumeister, et al., 2002), and to weaken one's self-regulation (Baumeister et al., 2005). An individual's understanding of his/her social situation, and the correspondent perceived effect of one's coping strategies, are in part influenced by one's cultural beliefs. For example, Uskul, Over, and Simpson (2014) found that individuals' coping strategies toward social exclusion varies by their diverse perceptions of the power of the perpetrators, which were in turn informed by their cultural background.

School-based bullying is a form of social exclusion manifest frequently among peers within groups of children and adolescents' (Killen \& Rutland, 2011), once again, broadly defined as aggressive behavior occurring in school that is targeted toward a victim (s) with malicious, repeated, and harmful 
intent (Olweus, 1993; Smith, 2004). School-based bullying has both short- long-term effects on targeted students' physical and mental wellness (Cheng et al., 2010; Wolke, et al.2013). Though school bullying is a common phenomenon, especially in the early adolescent years, recent research suggests significant differences exist in how it is perceived and dealt with across cultures (Elgar et al., 2015).

\section{3}

\section{Cultural Studies on Social Exclusion, Framed Empirically}

In China, empirical research on school-bullying, which began at around the turn into the 21st century (Smith, Kwak, \& Toda, 2016, pp. 113-132), has mostly been grounded in the Olweus "Norwegian based" conceptual framework (1993) rather than standing on its own cultural empirical lenses (Zhang, Chen, \& Chen, 2016). To localize foreign practices and initiate culturally appropriate research and practices on bullying in China, it is necessary to understand the various perceptions about bullying behaviors within the Chinese and western communities, particularly the perceived causes and advocated coping strategies.

Conventional comparative cultural frameworks suggest that while western culture tends to value an "independent" self, Chinese culture possesses strong collectivistic characters (Markus \& Kitayama, 1991), in particular a strong desire for and sense of community harmony (Cheng et al., 2010), thus focusing on locating the self within interdependent contexts, (Jen, 2013). These general distinctions on the conceptualizations of relationships between self and society can be seen as applied to theoretical definitions of bullying within Chinese and English discourses. "Bullying," as defined by The Ministry of Education of The People's Republic of China in Chinese is Qiling (欺凌) or baling (霸凌) puts an emphasis on the nature of the relationship rather on the frequency, as has been the case in Olweus (1993) Norwegian framework (Chen, et al., 2005; Cheng et al., 2010; Smith et al., 2016, pp. 133-150).

Different perceptions about the values of and relationship between self and society across eastern and western socialization practices also lead to culturally varied coping strategies for bullying (Lam \& Zane, 2004). When facing hypothetical peer conflicts, Asian American tended to propose internal change on self, while American participants were more likely to propose external change on the environment. Wright et al., (2016) for instance, found cultural variations among Chinese and American adolescents on their attribution on the cause of their own personal experiences of being bullied - Chinese adolescents were more likely to blame themselves than were American adolescents. 
In addition, Ma \& Bellmore (2016) have found that to cope with bullying Taiwanese students preferred seeking help from social relationship (teachers or friends), while American students tended to cope with the situation individually.

Relatedly, methodologically speaking, most traditional cross-cultural comparative studies on school-based bullying have employed "etic" approaches, using a priori, mostly western, theoretical frameworks to analyze cross-cultural responses (Woodside, 2010). Emic studies build conceptual frameworks from evidence rather than try to test the fit of evidence into already defined categories (Braun and Clarke, 2013; Morris, et.al., 1999). To understand comparatively Chinese and western perceptions and coping strategies around youth bullying, "emic" (inductive) analytical approaches are needed to examine the insiders' culturally normative view of bullying as it emerges from the qualitative data. Our study tries to fill in the gap by using emic analyses to interpret American and Chinese reviewers' perspectives about Wonder, a story about peer bullying in early adolescence and its impact beyond the victim to the victim' family. Wonder is easily accessible in print and film, both in China and the United States.

Ironically, we know very little, empirically speaking, about the impact of storytelling on the ethical or aesthetic sensitivities of individuals. Theoretically speaking, storytelling is viewed as a universally shared experience for humans across cultures and histories (Hsu, 2008). The connection between stories and the individuals who experience them is, according to literacy theorists, commonly constructed through the engaging aesthetic experiences that emerge from storytelling (e.g. Deniston-Trochta, 1998). In addition, individuals' understanding about fictional characters is seen as a natural extension of all people's understanding of themselves and others in the "real" world (Green, 2004).

That empirical research that exists does show that readers construct connections between themselves and the story to help them to reflect on personal experiences (Oatley, 1999), to develop social relational skills (Hsu, 2008), and to understand and cope with real-life struggles (Bowman, 2018). Practical cases also demonstrate that sharing self's and others' stories can support children to identify and cope with their own relational struggles (Salans, 2004). Relating individual experiences with the fictional characters and plots helps students feel safer and better able to cope with their own life challenges (Cordi \& Masturzo, 2013). 

Humanistic Story as an Opportunity for Empirical Research

The story Wonder, a popular children's (and young adult) novel (2/2012) and its movie adaptation (11/2017), depicts the struggles of August (Auggie) Pullman, a 10-year-old boy with Treacher Collins Syndrome - a craniofacial difference (deformity) that others might find upsetting to look at. The story tells what happens across one school year to Auggie, his family including his 14 years old sister, Via, and his new classmates, when he enrolls as a new fifth-grader in a private school, after being protectively homeschooled for the past five years of his life.

In this study, the protagonist, Auggie, represents individuals whose desires to be accepted and connected to peers is made challenging as they are singled out, or discriminated against, because of their physical, socio-cultural, or interpersonal differences. What kind of struggles do readers and movie reviewers believe Auggie, his families and friends face? What do these readers and viewers think are the appropriate strategies for Auggie and the other characters to cope with their struggles? Would people from western and eastern cultures making comments on this story via online review platforms have, on average, similar or different response patterns to these questions?

To better understand cultural variations in readers' and viewers' perspectives on Auggie's struggle to be accepted, we used a thematic topical analysis to explore the research question: How is Auggie's struggle to be accepted by his peers viewed in Chinese comments on Chinese websites, as compared with the English comments on North American websites? In addition, to understand reviewers' views on how Auggie's sister, Via, copes with the stresses and strains she experiences as a function of Auggie's condition, we used a discursive analysis of how the stories reviewers explained the ways in which they viewed Via's needs and responsibilities within the family. Using these two qualitative analytic lenses, we examined readers' and viewers' understandings of and perspectives on August's and Via's struggles that they, as on-line commenters, share through their unsolicited online posts on two websites. Given the popularity of Wonder, both in print and on film, and in both English- and Chinese-speaking communities, we also investigated what comments people from these two parts of the world, who have either read the book or have seen the movie, spontaneously made about aspects of the stories that motivate them to speak their minds toward their "online community". 
Specifically, while on-line commenters have license to comment on many story related topics, in our first analysis, after an open-coding phase of reading many reviewer comments for salient themes, we decided to examine only those reviewers' thoughts and opinions focused narrowly on our research question: How do reviewers make sense of Auggie's struggle for acceptance, and the ways he copes with the social challenges that other story characters pose, intentionally or otherwise? According to this criterion, $200^{1}$ comments were selected from the mostly used open-source story-reviewing websites, including one Chinese (Douban) and four English (Goodreads, Common Sense Media, Redeemed reader, and IMDb) among $160,0^{2}{ }^{2}$ reviews. We compared reviewers' comments about Auggie's struggle for acceptance as articulated by Chinese $(\mathrm{N}=100)$ and English reviewers $(\mathrm{N}=100)$, mainly anonymous and devoid of demographic or other identifying information, other than the website they select and the language they use to voice their comments. From a large pool of comments, and following a randomized sampling of over 1000 comments, purposeful sampling identified fifty of each cultural/language group to provide unsolicited reviews of the book; fifty of each reviewed the movie. To finalize our research question, our thematic analysis progressed in three steps:

1) Identified units of data. Comments that related specifically to the focal topic of the research question were isolated as the "unit of data." Although the comments covered a broad range of issues, our criterion in selecting units of data was by the reviewers' posts that specifically referred to Auggie's struggles to deal with the actions of his schoolmates. In all instances, the "unit of data" was the restricted comment on Auggie's struggle, while the emic "unit of analysis" (Boyatzis, 1998) within which each identified unit of data was located was the full individual on-line comment. (Therefore, there can be multiple units of data aligned with the emergent research question within each unit of analysis.)

2) Identified initial topical codes within analytic themes. Emic codes were developed upon the thematic analysis strategies prescribed by Boyatzis (1998) and Braun and Clarke (2006). The first author read through all comments to identify preliminary open codes, topics, and them possibly

1 Douban Book:50; Douban Movie: 5o; Goodreads (book review): 27; Common Sense Media (book review): 22; Redeemedreader (book review): 1; IMDb (movie review) : 50 .

2 Douban Book: 1924; Douban Movie: 104554; Goodreads (book review): 56356; Common Sense Media (book review): 258; Redeemedreader (book review): 5; IMDb (movie review) : 424 . (The date we counted the number: 04/07/2019). 
themes within which the codes can be nested. Subsequently, per guidelines specified by (Boyatzis, 1998), a 20\% random sample of the full sample (200 comments) was selected to develop inductive/ emic codes. Following initial code identification, analytical themes were identified, and conceptualizations of each theme were refined. Through this process, the initial codebook was developed to meet the criteria of five elements of good thematic coding outlined by Boyatzis (1998, p. 31): code titles, definitions, inclusion and exclusion descriptors, and exemplar text.

3) Refined Initial Codebook. In this process, the initial codebooks with the five elements (Boyatzis, 1998, p. 31) were refined by the first author's closely reading through the data, and the use of a multi-step inter-rater reliability (IRR) process. Then, the first author and her coder trained in thematic analysis coded a new random sample of 60 reviews in Excel. The two researchers then undertook a consensus inter-rater discussion to clarify disagreement and codes identified for further refinement. The codebook $^{3}$ was then revised, and a second IRR was conducted in qualitative analysis software using another random sample of 60 reviews.

\section{A Conceptual Framework and a Test of Inter-rater Agreement (Reliability)}

Through the process of iterative thematic qualitative analyses of all 200 selected full commenters, (28o total excerpts), we constructed a conceptual framework (Table 1) comprised as two primary emergent themes (primary dimensions), through which reviewers articulate their understanding on Auggie's struggle: 1) The Determinants (causes): the factors reviewers identify that animate Auggie's struggle. 2) The Coping Strategies: how reviewers describe or suggest that Auggie (might) cope with the interpersonal challenges he faces at school due to his "difference/abnormality".

For the theme Determinants, we reliably identified three sub-codes that emerged from the earlier emic analysis to provide texture, nuance, and withintheme variation Each sub-code suggested a distinct location of the reviewers' perceived causal determinants of Auggie's struggle: intrinsic struggles (Reviewers identify August's struggle as mainly located within himself, including intrinsic emotions, actions, behaviors and characteristics or traits); interactive struggles (Reviewers identify August's struggle as mainly located in the

3 The codebook is available on request from the first author. 
TABLE 1 Inter-rater reliability (Cohen's Kappa) of three codes for each of two Themes: Determinants Coping Strategies

\begin{tabular}{lcccccc}
\hline & Intrinsic & Interactive & Relational & $\begin{array}{c}\text { Personal } \\
\text { Efforts }\end{array}$ & $\begin{array}{c}\text { External } \\
\text { Support }\end{array}$ & $\begin{array}{c}\text { Collective } \\
\text { Solidarity }\end{array}$ \\
\hline Chinese & 1.00 & 0.88 & 0.85 & 0.83 & 0.38 & 0.49 \\
English & 0.90 & 0.92 & 1.00 & 0.91 & 0.90 & 0.81 \\
\hline
\end{tabular}

minds or hearts of the other book characters, including their intended harm or unintended reactions); relational struggles (Reviewers identify Auggie's and others' struggles as mainly located in the relationship between Auggie and other people in Auggie's social world. For examples, reviewers perceive others' struggles, e.g., his parents' or his sister's, as related to Auggie's struggle.)

Coping Strategies also comprised three topically different approaches. Reviewers tended to express variations in the range of Coping Strategies for Auggie to deal with his struggle to be: personal efforts (Reviewers identify the coping strategy as mainly initiated by Auggie himself, or the one who struggles); external support (Reviewers identify the coping strategy as mainly initiated by an agent other than Auggie, i.e., some-one, e.g., a friend in Auggie's social world.); collective solidarity (Reviewers identify the coping strategy as mainly the collective work of social agents which includes Auggie's efforts in connection to and together with the effort of others to deal with a shared struggle. (Specific examples of each of the three sub-codes are provided in the findings section to follow.

For analytic purposes, to remain in the coding system any codes developed or assigned to units of data must first achieve conceptually clear and acceptable inter-rater agreement (unblinded discussions among the team of coders), and then, adequate inter-rater reliability (blinded coding of identified units of coding). And the validity of the codes, primarily the interpretation or the understood meaning of the comments by the interpretive community, which in this case is our research lab, also must eventually reach consensus as to the code assigned to the comments.

We conducted the inter-rater reliability test using "Dedoose" software to calculate Cohen's Kappa. We selected $30 \%$ of the excerpts and set the Chinese and English data into two separated tests. The codebook the two coding partners (both are native Chinese speakers and proficient English speakers) used 
for the test was in English. The Cohen's Kappa reliability test results are presented in Table 1.

As indicated in the Table 1, we initially achieved high Kappa score for all codes, ranging from 0.81 to 1.oo, except for the codes 'External Support' and 'Collective Solidarity' under Chinese reviews, with relatively low Kappa scores of 0.38 (external support) and 0.49 (collective solidarity). We reviewed and discussed the excerpts about which the two coding partners disagreed for each of these two "codes." We found that the two coding partners had different understandings of the definition of the two codes when applying the codebook as written in English, i.e. as translations of the Chinese reviews. That is, the two Chinese/English fluent coding partners identified the definition of the "external" and "collective" differently in an English language context.

To improve the inter-rater reliability, we then went back to a process to understand and improve inter-rater agreement. We reviewed the English versions of the excerpts under these two codes, back translated the excerpts into Mandarin, and found the two coding partners had high agreement on them when the comments were coded in Mandarin. In response to this situation, we constructed a codebook in Mandarin Chinese and the two coding partners reached the consensus on all the 'Collective Solidarity' and 'External Support' codes as found in the Chinese excerpts. In the second round of reliability test on these two codes in Chinese, we randomly selected 30 percent of excerpts, including some different from the first round of reliability test, and achieved the kappa score of 1 for both the two codes. ${ }^{4}$

Preliminary Findings Memo: a Code Book, A Conceptual Framework, and a Chi Square Analysis

Once we had established the inter-rater reliability of the two primary thematic codes and their correspondent three sub codes for each, we compared and contrasted the distribution of these six sub-codes as they emerged within the

4 Throughout the process of constructing the conceptual framework and its alignment with the operational code book used for purposes of data collection, we achieve a shared understanding of what the various reviewers meant by their comments on "Auggie's struggle," both with respect to its determinants and the ways the struggle can be coped with by Auggie (and subsequently by the other characters in the story). We gained confidence in our inferences and interpretations based upon the inter-rater agreement and inter-rater reliability of our team's coding of the corpus of comments in the sample. (Both coders were fully competent in both written and spoken English and Mandarin.) 
TABLE 2 Distribution of excerpts under each primary theme and in each subordinate code by language used and media reviewed

\begin{tabular}{lcccc}
\hline & $\begin{array}{c}\text { English Book } \\
\text { Review }\end{array}$ & $\begin{array}{c}\text { English Movie } \\
\text { Review }\end{array}$ & $\begin{array}{c}\text { Chinese Book Chinese Movie } \\
\text { Review }\end{array}$ & $\begin{array}{c}\text { Review } \\
\text { Determinants }\end{array}$ \\
Intrinsic & 38 & $\mathbf{3}^{8}$ & $\mathbf{2 0}$ & $\mathbf{2 2}$ \\
Interactive & 5 & 10 & 4 & 5 \\
Relational & 23 & 13 & 12 & 11 \\
Coping Strategy & 10 & 15 & 4 & 6 \\
Personal Efforts & 40 & 27 & 54 & 41 \\
External Support & 15 & 10 & 14 & 10 \\
Collective Solidarity & 10 & 10 & 13 & 9 \\
& 15 & 7 & 27 & 22 \\
\hline
\end{tabular}

Note: Although the analysis encompasses 200 units of analysis, i.e., 200 individuals, some includes multiple units of data, that are excerpts that each has its' own distinct thematic code. Therefore, the full total sample of excerpts that were coded numbered 280 .

full sample in both of the two media forms (i.e. book and film reviews) and across the two cultural samples (i.e. Chinese vs. English speaking websites). Table 2 below presents a count of the focal thematic thoughts that the Chinese and the English reviewers expressed about the protagonist's struggle. Responses are also disaggregated for comments by the book reviewers and the movie reviewers within each of the two cultural language samples.

As we did not find any striking differences when using the code book to compare comments on the story presented across media, i.e., book and movie, either in the construction of the conceptual framework, thematically speaking, or in the distribution of code usage, numerically speaking, for the purposes of this analysis, we combined the responses of the reviewers in each of these two media samples for purposes of comparative (statistical) analysis. ${ }^{5}$

Although there were similar numbers of total responses coded from the English and Chinese samples, of the 143 responses in English, 53 percent ( 76 out of 143) were coded as "Determinant" and 47 percent were coded as "Coping Strategies". Among the responses in Chinese, only 31 percent (42 out of 137) were coded as "Determinant", and nearly 70 percent were "Coping

5 Analyses of similarities/differences in reviews across media within which the story was told by sub-codes were cursorily examined due to the limits of the sample size and of any salient demographic indicators. 
Strategies." A Chi Square analysis of the distribution of all the excerpts (units of data) in the Chinese and English samples, and the two primary themes, Determinants (causes) and Coping Strategies each sample favored indicated that the differences are large enough to be statistically significant $(\mathrm{P}<0.0001){ }^{6}$

10

What do Reviewers Really Mean When They Comment on Specific Determinants and Coping Strategies: some Descriptive Examples

A qualitative topical analysis of the prevalence of codes across the two different groups of reviewers (Chinese-speaking vs. English-speaking) provides some more granular interpretations of the statistical findings. For one example, with respect to a comparison within the Determinant theme, the excerpts (units of coding) of the English-speaking reviewers, as compared to the Chinesespeaking reviewers, suggest a relatively greater emphasis on comments that located the determinants of Auggie's struggle, that is, an expressed interest in understanding or explaining its causes as intrinsic. (Units of coding 'intrinsic determinant' in English = 15, in Chinese 9). An example of an 'intrinsic determinant' excerpt from an English reviewer is:

This book is about a young boy named August Pullman who was born with a facial disability and is embarrassed of how he looks.' It does get you welling up and angry, the kid is trying to overcome his shyness and trying to brave his first time at school with a facial deformity.

In contrast, as compared to the English reviews, not only did the Chinese reviews tend to more often make recommendation as to various coping strategies that might provide a direct resolution of the struggle, within the theme of coping mechanism, not surprisingly, there also was a larger proportion of comments addressed or suggested the Collective Solidarity code ('English collective solidarity responses $=22$; in Chinese $=49$ collective solidarity responses). A Mandarin translated example of 'Collective Solidarity' coping strategy excerpt from Chinese reviewer is:

6 We applied various methods of Chi Square statistical analyses to these distributions; Likelihood Ratio, Continuity Adjusted, Mantel-Haenze. Given the small numbers of coded excerpts for each of the sub-codes, we did not comparatively analyze them across language groups statistically, although an inspection of the actual comments suggests some possible meaningful variations we will discuss in the context of our narrative analysis. 
The Pullman family, including Daisy (the dog), was a depiction of a family structure that was imperfectly perfect in how it handled its growing pains, by accomplishing the best and perhaps only action necessary to overcome tribulation; be together.

In a second kind of in-depth exploratory qualitative analysis, we focused on whether there were possible differences in ways the two cultural groups "positioned" actors, even when the excerpted comments from both language groups were assigned to the same sub-code. For example, within the Coping theme, we compared the Personal Effort coping sub-code as it was used by Chinese (24 instances) and English (25 instances) online comments on Auggie's struggles to see if the groups attributed different kinds of personal efforts as ways to cope with Auggie's struggle. Even though the two language groups used about the same number comments reliably coded as Personal Effort, a discursive analysis (Haste, 2013) of all the comments so coded thematically, suggested two possibly very different cultural interpretations on the kinds of personal efforts type recommended to be taken.

We heard what we called a Self-transforming personal effort narrative that was more often suggested by (Chinese-speaking) online reviewers who tended to perceive the effective solutions for social conflicts by strengthening the self. In contrast, when facing social challenges such as Auggie's, we heard Othertransforming types of personal effort, i.e., making efforts to invoke external changes in others, e.g., the perpetrators, more often suggested by Englishspeaking online reviewers. Table 3 presents two exemplar comments, one in Chinese and one in English, both of which was assigned the "Personal Effort" code.

TABLE 3 Examples of different cultural narratives within the code "Personal Effort"

Prototype Change/ strengthen the Self Coping comments.
Prototype Change/ transform the Other Coping comments.

3.1. “也许成长就是在不断的自我怀 3.2. “This book teaches an amazing lesson of 疑, 自我完善, 逐渐变成一个勇敢自 being inclusive and not letting what others say 信的成年人的过程。”

[May be growing up is the process to keep questioning yourself and improving yourself. By doing so, you will define who you are, and that only you define yourself. Even though Auggie was severely bullied, he did not let others get to him and he still believed in himself, and that is an example become a brave and confident adult.] of an amazing role model in a book." 
The examples in Table 3 demonstrate these "sub-types" do not essentialize, but deepen our understanding of the two cultural orientations. Englishspeaking reviewers focus on Auggie's efforts to influence the antagonists at the school, e.g. by taking actions that refute others' opinion is exemplified in comment 3.2 in Table 3. But English-speaking reviewers also tended to phrase personal effort in terms of Auggie's character, e.g., his struggle to be brave. An example is 'I loved that August stayed strong when Jack was talking.' Chinese reviewers, on the other hand, while often mentioning the importance of Auggie's character development, do so in a meaningfully different way by focusing more on Auggie's need to develop a strong inner character; not so much by his striking outward, but instead by looking inward to find inner strength (example 3.1 in Table 3).

This preliminary hunch, or evidence, aligns with previous empirically based cultural findings on individuals' preferred coping strategies among Asian and United States participants on school bullying, (Lam \& Zane, 2014; Wright et al., 2016) and is supported by the early important cross-regional cultural framework (Markus \& Kitayama, 1991) that comparatively described conceptualizations on self and social relationship between Eastern and Western societies. Self- and Other-Transforming orientations have also been validated from a cognitive developmental theoretical perspective. (Selman et al., 1986). This latter within-culture (English/U.S.A.) analysis highlights that such variations in personal effort within a single culture's normative framework can also be considered more or less mature, but cannot be culturally essentialized.

\section{Analysis 2: a Discursive Analysis of Via's Relational Struggle Within the Family}

In our analysis focused on Auggie's struggle to be accepted by his peers we found that it was not uncommon for reviewers to shift their attention and discussion within their complete comments (the unit of analysis) from August's struggle to the struggles of his family members, especially his elder sister, Via. We discerned that although Via's struggles (needs, desires) were particular to Auggie's condition in this story, they were also representative of the everyday needs of adolescents independent of the particulars of this story. Using the same data set, therefore, we compared the English-speaking and Chinesespeaking reviewers reasoning when they spontaneously included Via's struggle in their comments. As there were fewer comments in the set on this topic, we used a line by line narrative analysis (Bruner, 1991) of the reviewers' whole comment (the full unit of analysis) rather than the unit of coding approach 
(excerpts within the full comment) undertaken for the thematic analysis. We examined the meaning of the comments in the two languages, focusing on what we identified as commenters' narratives about "Via's relational struggle within the family" (Abell, Stokoe, \& Billig, 2001).

During the process, we paid close attention to the meaning of comments in terms of who is Via, and what Via was "doing" or trying to achieve within the family. We discerned two dimensions of reviewers' conceptualization of Via in their narratives: 1) Protect Other (Auggie): Via is a persevering and caring elder sister; she has been enduring but doing something nice for Auggie, etc. and 2) Protect Self: Via is vulnerable; she has been seeking support from her mother, remembering the support of her (deceased) grandmother.

TABLE 4 Examples of variations by language group in the self's position and cultural values: The case of Via's struggle English Commenters Mandarin Commenters

\section{Protect Self: Independent construal Protect Other: Interdependent construal orientation (dominant narrative of orientation (dominant narrative of the the English-speaking reviewers) Chinese-speaking reviewers)}

4.1.1 "Basically, she has lived in her

4.2.7. “我心疼维娅, 她是姐姐, 那么懂事, 可 little brother's shadow all over her life. I 她也是孩子,她也需要爸爸妈妈的矢爱, found it rather sad to read her thoughts. 而她却甘愿做围绕在奥吉身边的行星” How she always had to fend herself when her littler brother was the center of attention and how she sometimes [Translation: Ifeel sorry for Via, She is the older sister. She understands her family's needs hates herself to take that attention away also a kid who needs parents' care and love. from him." But she is still willing to live her life around 4.1.2. "Though she loves him, she can't Auggie, being a satellite orbiting around help feeling pushed aside by his Auggie.] never-ending physical and emotional needs."

4.1.3. "Here we have this girl who loves her little brother and wants to protect 4.2.2. “懂事的姐姐爱弟弟但也会委屈” [Translation: The sister understands her role and responsibility, but she is still suffering] 4.2.3. “他的成长离不开父母全心全意的 him and help him and be there for him 付出和牺牲 也离不开姐姐的无声无息的 anytime he needs her but we also see 隐忍和懂事” (He(Auggie)'growth relies on how badly she wants her parents to pay more attention to her and her the full dedication and carification of his parents, and the silent tolerance and underproblems and that's normal and right." standing of his sister. ) 
Though both English and Chinese reviews connected Via's struggle to Auggie's suffering, English-speaking reviewers tended to position Via more often in the "Protect Self" dimension: attributing Via's struggle to her personal need for attention and care from the family.

These comments tended to see Via's "legitimate" need to be satisfied as being harmed by Auggie's "special" needs. (example 4.1.2). Chinese reviewers, on the other hand, tended to focus more on the "Protect Other" dimension: They viewed Via's struggle as the challenge she faces in caring for and supporting Auggie as a member of the family, whether that support is a function of her inherent caring nature or a function of her family obligations and duties (example 4.2.1). The Chinese-speaking reviewer here positioned Via in her familial role as an elder sister so as to emphasize her actions towards Auggie: she "understands the needs" and "is willing to live her life around Auggie". What Via "wants" as an independent being is not explicitly mentioned here unlike what we found in English-speaking reviews (example 4.1.3). Moreover, while Chinese Speaking reviewers are aware of or acknowledge Via's need or expectation for her parents' attention and love, it is often viewed as not a fully accountable claim.

The exploratory evidence of readers' diverse interpretations on Via's story raises a question - why would Chinese- and English- speaking reviewers perceive the determinant of Via's struggle differently? Do English- and Chinese- speaking commenters position the relation of self to other in Via's social situation in accordance with how they weigh the values of the larger society and its norms and expectations differently? Do English-speaking readers more often tend to position the self as at the center among social relationship, and emphasize individual's needs, while Chinese readers might tend more often to others' needs in the context of normatively prescribed social roles and expectations such as the one depicted in Wonder?

In this analysis, English- and Chinese- speaking commenters read, or viewed, the same story, but construct different meanings to the plot and characters., English comments tended to attribute Via's struggle to her need/ expectation to be loved by her parents, and her need for connection not being satisfied, especially because of her parents' lack of attention and caring. For example, the comments of reviewer 4.1.1 reconstructed Via's experience by highlighting her 'wanting to take the attention' from their family'. Via herself is perceived accountable for her struggle in a context, but she is also positioned 
as being vulnerable because 'she lived in her little brother's shadow'. Chinese commenters recognized Via's desire or need as an adolescent for more direct support from her parents, but more often tended to identify as more important Via's struggle to be her striving to meet the family's needs and take the responsibility as an elder sister (examples 4.2.1-3).

These variations among English and Chinese comments on interpreting the nature of Via's struggle supports previous conceptual frameworks that describes two cultural narratives of the responsibilities and values of the self: an independent orientation as the "distinguished universe" (Geertz, 1975) that achieves self-values by demonstrating self's uniqueness; and a narrative of interdependent self-construal often described as an interconnected social component that perceives self-values as achieving social responsibility and needs (Kitayama, Karasawa, Curhan, Ryff, \& Markus, 2010).

\section{A Summary of Our Study and Practical Implications: bridging Cultures and Closing the Circle}

We selected a recently published (2012) and film-produced (2017) popular fictional story, Wonder - which depicts the experiences across a school year of August (Auggie) Pullman, a middle school student, born with a genetic facial deformation, as he struggles with social exclusion, especially school-based relational bullying. The story also depicts Auggie's elder sister Via's own perspective, more specifically, the struggles she faces in trying to meet her own needs for support within their family as a consequence of Auggie's challenges. With our interest in the variations in audiences' responses to the story as told both across media (book and film), and across cultures (Chinese and English bookand movie-review websites), we investigated online reviewers' epistemic understandings (Elgin, 2006, 2017) of Wonder through analyzing the comments from selected users of popular Chinese and English online platforms.

Based on our analyses, we constructed a conceptual framework that contains two "emergent orienting" lenses: the reviewers' understanding of the determinants of Auggie's and Via's struggles, and of the possible coping strategies reviewers perceived as available to Auggie and Via, (and other fictional characters in Wonder) to deal with their personal struggles. This framework aligns with previous empirical studies on the thoughts and the actions people voluntarily share about interpersonal struggles, specifically the causes and effects of school bullying (Camodeca \& Goossens, 2005; Skrzypiec, et al., 2011; Ferrans, Selman, \& Feigenberg, 2012; Weinstein, et al., 2015; Evans, Cotter, \& Smokowski, 2017). 
Analysis 1 suggested that comparatively, Chinese-speaking reviewers tend to more often focus on the value of solving the problem, and in doing so, articulating the corresponding ways a victim of bullying or neglect can be or become both strong and interdependent, whereas English-speaking reviewers more often tend to emphasize exploring and understanding the causes of the relational problems and tend to suggest independence-oriented ways of solving the social problem by changing the actions of others. It also revealed different meanings given to various coping strategies. A striking example is how the idea of "personal effort" as a coping strategy meant something different, if not distinct, to members of each language group. Analysis 2 suggests meaningful differences across Chinese- and English-speaking reviewers in both their perceptions of and judgements about how Via, Auggie's sister, should deal with her feelings of being unsupported in her relational struggles within her family.

In this final section, we will briefly propose two practical implications inspired by these preliminary hunches. Firstly, storytelling can be a powerful tool for educators within each cultural in helping students cope with their struggles in life. Since the stories not only present vivid incident of social exclusion, but also the characters' reactions and corresponding plot development, young readers of the stories could learn from the coping strategies used by the characters as they are engaged in looking at their own life challenges (Allen, Krebs, \& Palgrave, 2007). An example from our data is: 'I relive moments from this book, and it makes me really appreciate the importance of true beauty and human goodness and those who are strong enough to attain it. True beauty is earned, we have to fight every day to be beautiful inside and I cringe at how easy it is to be the opposite - ugly and cruel.'The reviewer here talked about the coping strategy of building beauty inside as gleaned from the book to cope with one's own everyday struggles.

In this analysis, we found that reviewers from Eastern and Western language cultures not only emphasize different aspects of the two social problems (bullying and need for support within the family), e.g. seeking the cause and seeking the solution, they very well may use different approaches when talking about the coping strategies seen as preferable in the problem's resolution: Chinese reviewers tend to talk more about the coping strategy of seeking for collective solidarity while English reviewers tend to adopt the coping strategy of initiating actions to change others (bullies)' opinions and behaviors. From theses insights, we suggest practices considering individual's cultural preference and the situational climate. Educators working with Chinese students could focus more on seeking 'social support' from parents, friends or teachers as the way to cope with social exclusion. Educators working with English students 
could provide more suggestions on handling confrontation with peers, especially those peers who are actively engaged in bullying or ostracism.

Second, these analyses suggest opportunities to utilize storytelling for intercultural awareness education as well. One example would be to make use of the cultural variations in perspectives on both Auggie's and Via's struggle, both with students within and between cultures. While the differences across cultures are striking, there lies underneath the variations in perspective common themes which bends toward the universal, resonating for educators or caregivers across cultures. For instance, while Auggie's struggle for acceptance that are associated with his immutable physical differences is heart-wrenching and salient in the book, it is, however, an uncommon occurrence, albeit one that speaks to differences of many sorts. The theme that speak to nearly every family and culture, is embedded in Via's struggle. She is drawn into her family struggle and has been ignored by her mother (due to Auggie's needs). Compared to Auggie's struggle, in that sense, Via's feelings of exclusion, even though perceived differently in our comparison of two cultures, can be considered more commonplace around the world.

Finally, we wish to briefly point out the future opportunities to implement these two practical aims through what we call cross-media storytelling. In this study, we drew on the online comments of reviewers in two cultures who experienced the story, Wonder, either through text or film. Elsewhere, we have written about both the theoretical foundations (Selman, Testa, \& He, in press) and the practical applications of an approach to youth development using Wonder as a case of how to use cross-media applications Pilot work suggests the educational value of watching a film of a story that also has been read, not only for literacy development and disciplinary learning, e.g. historical understanding, but also for the practicing of a "foreign language", a primary cultural experience (Wang et al., 2020).

We close with a return to our discussion on our study of culture with the story Wonder, which is not only about "social exclusion" but also "friendship" and how to cope with differences. In Wonder, at the psychological level, those classmates who see Auggie only by his face, born of deformity, ignore, or are unaware of, his humor, smartness, and the normality of his struggle. At the sociological level, as discussed previously, seeing or being aware of differences across cultures enables us to situate educational practices that respect an individuals' cultural needs and preferences. Acknowledging the similarities might be the first step to appreciate the differences, comparative storytelling, told across media and cultures, used wisely in diverse cultural context, might be an important and valuable approach. We need now to carefully curate additional stories, coming from each cultural tradition. 


\section{References}

Abell, J., Stokoe, E. H., \& Billig, M. (2000). The discursive (re)construction of events. In Andrews, M., S. Day Sclater, S., Squire, C., \& Treacher, A. (Eds.), Lines of narrative: psychosocial perspectives (pp. 180-192). London: Routledge.

Arseneault, L., Bowes, L., \& Shakoor, S. (2010). Bullying victimization in youths and mental health problems: 'Much ado about nothing'? Psychological Medicine, 4o(5), 717-729. https://doi.org/10.1017/Soo33291709991383.

Baldry, A. C. (2004). The impact of direct and indirect bullying on the mental and physical health of Italian youngsters. Aggressive Behavior, 3o(5), 343-355. https:// doi.org/10.1002/ab.20043.

Baumeister, R., DeWall, C., Ciarocco, N., Twenge, J., \& Devine, Patricia. (2005). Social Exclusion Impairs Self-Regulation. Journal of Personality and Social Psychology, $88(4), 589-604$.

Baumeister, R., Twenge, J., Nuss, C., \& Devine, Patricia. (2002). Effects of Social Exclusion on Cognitive Processes: Anticipated Aloneness Reduces Intelligent Thought. Journal of Personality and Social Psychology, 83(4), 817-827.

Bowman, R. (2018). Teaching and Learning in a Storytelling Culture. The Clearing House: A Journal of Educational Strategies, Issues and Ideas, 91(3), 97-102.

Boyatzis, R. E. (1998). Transforming qualitative information: Thematic analysis and code development. SAGE. Sage Publication, London.

Braun, V., \& Clarke, V. (2006). Using thematic analysis in psychology. Qualitative research in psychology, 3(2), 77-101.

Bruner, J. (1991). The narrative construction of reality. Critical inquiry, 18(1), 1-21.

Cambodia, M., \& Goossens, F. A. (2005). Children's opinions on effective strategies to cope with bullying: The importance of bullying role and perspective. Educational research, 47(1), 93-105.

Cordi, K., \& Masturzo, K. (2013). Using literature and digital storytelling to create a safe place to address bullying. Voices from the Middle, 20(3), 21.

Chen, X., Cen, G., Li, D., \& He, Y. (2005). Social Functioning and Adjustment in Chinese Children: The Imprint of Historical Time. Child Development, 76(1), 182-195. Retrieved from Jstor.

Chen, X., Huang, X., Chang, L., Wang, L., \& Li, D. (2010). Aggression, social competence, and academic achievement in Chinese children: A 5-year longitudinal study. Development and Psychopathology, 22(3), 583-592. https://doi.org/10.1017/Sog5457 9410000295 .

Cheng, Y., Newman, I. M., Qu, M., Mbulo, L., Chai, Y., Chen, Y., \& Shell, D. F. (2010). Being Bullied and Psychosocial Adjustment Among Middle School Students in China. Journal of School Health, 8o(4), 193-199. https://doi.org/10.1111/j.1746-1561.2009 .00486.x. 
Deniston-Trochta, G. M. (1998). The meaning of storytelling as pedagogy. Visual Arts Research, 24(2), 27-32.

Elgar, F. J., McKinnon, B., Walsh, S. D., Freeman, J., D. Donnelly, P., de Matos, M. G., ... Currie, C. (2015). Structural Determinants of Youth Bullying and Fighting in 79 Countries. Journal of Adolescent Health, 57(6), 643-650. https://doi.org/10.1016/j .jadohealth.2015.08.007.

Elgin, C., (2006) From Knowledge to Understanding. Ed. Stephen Hetherington, Epistemology Futures. Oxford: Clarendon Press, 199-215.

Elgin, C. (2017) True Enough, MIT Press, Cambridge, Mass.

Evans, C., Cotter, B., Smokowski, R. (2017). Giving victims of bullying a voice: a qualitative study of post bullying reactions and coping strategies. Child and Adolescent Social Work Journal. 34(6), 543-555.

Ferrans, S., Selman, R. L., \& Feigenberg, L. (2012) Rules of the culture and personal needs: Witnesses' decision-making process to deal with situations of bullying in middle school. Harvard Educational Review, 82, 445-470.

Geertz, C. (1975). On the Nature of Anthropological Understanding: Not extraordinary empathy but readily observable symbolic forms enable the anthropologist to grasp the unarticulated concepts that inform the lives and cultures of other peoples. American Scientist, 63(1), 47-53. Retrieved from JSTOR.

Green, Melanie C. (2004). Transportation In to Narrative Worlds: The Role of Prior Knowledge and Perceived Realism. Discourse Processes: A Multidisciplinary Journal, $38(2), 247-266$.

Haste, H. (2013). Culture, Tools and Subjectivity: The (Re) Construction of Self. In T. Magioglou (Ed.), Culture and Political Psychology (pp. 27-48). Information Age.

Hsu, J. (2008). The Secrets of Storytelling. Scientific American Mind, 19(4), 46-51.

Jen, G. (2013). Tiger Writing: Art, Culture, and the Interdependent Self. Cambridge, Massachusetts: Harvard University Press.

Kanetsuna, T., Smith, P. K., \& Morita, Y. (2006). Coping with bullying at school: children's recommended strategies and attitudes to school-based interventions in England and Japan. Aggressive Behavior, 32(6), 570-580. https://doi.org/10.1002/ ab.20156.

Killen, M., \& Rutland, A. (2011). Children and social exclusion: Morality, prejudice, and group identity. John Wiley \& Sons.

Kitayama, S., Jackendoff, R., Lambert, W., Duncan, R., Lucy, J., Markus, H., ... Weller, R. (n.d.). The Four Elementary Forms of Sociality: Framework for a Unified Theory of Social Relations.

Kitayama, S., Karasawa, M., Curhan, K. B., Ryff, C. D., \& Markus, H. R. (2010). Independence and Interdependence Predict Health and Wellbeing: Divergent Patterns in the United States and Japan. Frontiers in Psychology, r. https://doi.org/10.3389/ fpsyg.2010.00163. 
Lam, A. G., \& Zane, N. W. S. (2004). Ethnic Differences in Coping with Interpersonal Stressors: A Test of Self-Construals as Cultural Mediators. Journal of Cross-Cultural Psychology, 35(4), 446-459. https://doi.org/10.1177/0022022104266108.

Ma, T.-L., \& Bellmore, A. (2016). Connection or independence: Cross-cultural comparisons of adolescents' coping with peer victimization using mixed methods. Journal of Cross-Cultural Psychology, 47(1), 109-130. https://doi.org/10.1177/0022022115605386.

Markus, H. R., \& Kitayama, S. (1991). Culture and the self: Implications for cognition, emotion, and motivation. Psychological Review, 98(2), 224-253. https://doi .org/10.1037/0033-295X.98.2.224.

Matsumoto, D. (1999). Culture and self: An empirical assessment of Markus and Kitayama's theory of independent and interdependent self-construal. Asian Journal of Social Psychology, 2(3), 289-310. https://doi.org/10.1111/1467-839X.ooo42.

Morris, M., Leung, K., Ames, D., \& Lickel, B. (1999). Views from inside and outside: Integrating emic and etic insights about culture and justice judgment. Academy of Management Review, 24(4), 781-796.

Oatley, K. (1999). Meetings of minds: Dialogue, sympathy, and identification, in reading fiction. Poetics, 26(5), 439-454.

Olweus, D. (1993). Bullying at School: What We Know and What We Can Do (Vol. 12). Somerset: John Wiley \& Sons.

Riva, P., Eck, J., \& Riva. (2016). Social exclusion. Berlin, Germany: Springer.

Salans, M. (2004). Storytelling with children in crisis: Take just one star-How impoverished children heal through stories. Jessica Kingsley Publishers.

Selman, R. L., Beardslee, W., Schultz, L, Krupa, M., and Podorefsky, D. (1986) Assessing adolescent interpersonal negotiation strategies: Toward the integration of structural and functional models. Developmental Psychology, 22(4), 450-459.

Selman, R. L. (2003). Promotion of Social Awareness: Powerful Lessons for the Partnership of Developmental Theory and. Retrieved from http://muse.jhu.edu/book/10884.

Selman, R. L. and Testa, R. M. (2019). Twice-Told Tales: Re-thinking Books, Movies and Teaching Through Close Cross-Media Comparisons. HgSE Alumni Bulletin, September, 2019 .

Selman, R. L. Testa, R., \& He, H. (in press) The Art and Science of Humanistic Tales Twice-told: Opening Minds Aesthetically, Academically, and Ethically. In, Riggs, W. Ed. Open-mindedness \& Perspective, Oxford.

Skrzypiec, G., Slee, P., Murray-Harvey, R., \& Pereira, B. (2011). School bullying by one or more ways: Does it matter and how do students cope? School Psychology International, 32(3), 288-311.

Smith, P. B., \& Bond, M. H. (1993). Social Psychology Across Cultures: Analysis and perspectives. Hemel Hempstead, England: Harvester Wheatsheaf.

Smith, P. K. (2004). Bullying: Recent Developments. Child and Adolescent Mental Health, 9(3), 98-103. https://doi.org/10.1111/j.1475-3588.2004.00089.x. 
Smith, P. K., Kwak, K., \& Toda, Y. (2016). School Bullying in Different Cultures. Cambridge University Press.

Uskul, A., Over, H., \& Simpson, Jeffry A. (2014). Responses to Social Exclusion in Cultural Context: Evidence From Farming and Herding Communities. Journal of Personality and Social Psychology, 106(5), 752-771.

Wang, L., Zhang, W., Selman, R. L. \& Testa, R. M. (2020). Educator Resource for Wonder. https://xmedia.gse.harvard.edu/.

Weinstein, E. C., Selman, R. L., Thomas, S., Kim, J. E., White, A. E., \& Dinakar, K. (2016). How to cope with digital stress: The recommendations adolescents offer their peers online. Journal of Adolescent Research, 31(4), 415-441.

Wolke, D., Copeland, W. E., Angold, A., \& Costello, E. J. (2013). Impact of Bullying in Childhood on Adult Health, Wealth, Crime, and Social Outcomes. Psychological Science, 24(10), 1958-1970. https://doi.org/10.1177/0956797613481608.

Woodside, A. G. (2010). Case study research: Theory, methods and practice. Emerald Group Publishing.

Wright, M. F., Yanagida, T., Ševcíková, A., Aoyama, I., Dedková, L., Machácková, H., ... Shu, C. (2016). Differences in Coping Strategies for Public and Private Face-to-Face and Cyber Victimization among Adolescents in Six Countries. International Journal of Developmental Science, 1o(1), 43-53. (Ios Press. Nieuwe Hemweg 6B, Amsterdam, 1013 B G, The Netherlands. Tel: +31-20-688-3355; Fax: +31-20-687-0039; e-mail: info@ iospress.nl; Web site: http://www.iospress.nl). 